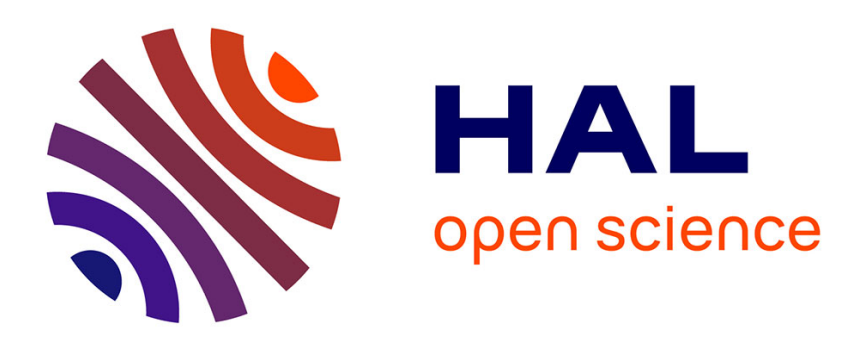

\title{
Neural activity of heterogeneous inhibitory spiking networks with delay
}

\author{
Stefano Luccioli, David Angulo-Garcia, Alessandro Torcini
}

\section{To cite this version:}

Stefano Luccioli, David Angulo-Garcia, Alessandro Torcini. Neural activity of heterogeneous inhibitory spiking networks with delay. Physical Review E , 2019, 99 (5), 10.1103/PhysRevE.99.052412 . hal-03091610

\author{
HAL Id: hal-03091610 \\ https://hal.science/hal-03091610
}

Submitted on 31 Dec 2020

HAL is a multi-disciplinary open access archive for the deposit and dissemination of scientific research documents, whether they are published or not. The documents may come from teaching and research institutions in France or abroad, or from public or private research centers.
L'archive ouverte pluridisciplinaire HAL, est destinée au dépôt et à la diffusion de documents scientifiques de niveau recherche, publiés ou non, émanant des établissements d'enseignement et de recherche français ou étrangers, des laboratoires publics ou privés. 


\title{
Neural activity of heterogeneous inhibitory spiking networks with delay
}

\author{
Stefano Luccioli, ${ }^{1}$ David Angulo-Garcia, ${ }^{2}$ and Alessandro Torcini ${ }^{3,1}$ \\ ${ }^{1}$ CNR-Consiglio Nazionale delle Ricerche-Istituto dei Sistemi Complessi, via Madonna del Piano 10, 50019 Sesto Fiorentino, Italy \\ ${ }^{2}$ Grupo de Modelado Computacional-Dinámica y Complejidad de Sistemas. Instituto de Matemáticas Aplicadas. \\ Universidad de Cartagena. Carrera 6 \# 36 - 100, Cartagena de Indias, Colombia \\ ${ }^{3}$ Laboratoire de Physique Théorique et Modélisation, Université de Cergy-Pontoise, CNRS, UMR 8089, 95302 Cergy-Pontoise cedex, France
}

(Received 11 February 2019; revised manuscript received 18 April 2019; published 23 May 2019)

\begin{abstract}
We study a network of spiking neurons with heterogeneous excitabilities connected via inhibitory delayed pulses. For globally coupled systems the increase of the inhibitory coupling reduces the number of firing neurons by following a winner-takes-all mechanism. For sufficiently large transmission delay we observe the emergence of collective oscillations in the system beyond a critical coupling value. Heterogeneity promotes neural inactivation and asynchronous dynamics and its effect can be counteracted by considering longer time delays. In sparse networks, inhibition has the counterintuitive effect of promoting neural reactivation of silent neurons for sufficiently large coupling. In this regime, current fluctuations are on one side responsible for neural firing of subthreshold neurons and on the other side for their desynchronization. Therefore, collective oscillations are present only in a limited range of coupling values, which remains finite in the thermodynamic limit. Out of this range the dynamics is asynchronous and for very large inhibition neurons display a bursting behavior alternating periods of silence with periods where they fire freely in absence of any inhibition.
\end{abstract}

DOI: 10.1103/PhysRevE.99.052412

\section{INTRODUCTION}

Despite the fact that inhibition emerges only at later stages of development of the brain [1], its role is fundamental for a correct and healthy functioning of the cerebral circuits. In the adult brain, the majority of neurons are excitatory, while only $15-20 \%$ has been identified as inhibitory interneurons. However, this limited presence is sufficient to allow for an overall homeostatic regulation of global activity in the cerebral cortex and at the same time for rapid changes in local excitability, which are needed to modify network connections and for processing information [2].

The role of inhibition in promoting brain rhythms at a mesoscopic level, in particular in the beta $(12-30 \mathrm{~Hz})$ and gamma $(30-100 \mathrm{~Hz})$ bands, has been clearly demonstrated in experiments and network models [3,4]. In particular, recent optogenetic analysis in vivo have shown the relevance of specific interneuron inhibitory populations: namely, parvalbumin-positive interneurons are essential for gammarhythm generation in the cortex [5,6]; while somatostatinpositive and parvalbumin-positive interneurons orchestrate sensory induced beta and gamma cortical oscillations [7]. On one hand, synaptic inhibition enhances short-timescale correlations in the spiking activity by promoting the generation of oscillations and pairwise synchrony between excitatory neurons. On the other hand, inhibition suppresses long-timescale correlations, such as those due to noise, potentially favoring an enhancement of neural encoding [8].

This justifies the interest for the dynamics of purely inhibitory neural networks and in particular for the emergence of collective oscillations (COs) in these systems. COs have been usually reported for networks presenting either a time delay in the transmission of the neural signal or a finite synaptic timescale. An interesting analogy can be traced between the dynamics of inhibitory networks with delay and instantaneous synapses, and that of circuits where the postsynaptic potentials (PSPs) have a finite duration and an instantaneous synaptic transmission. In particular, in Ref. [9] it has been shown that in homogeneous fully coupled networks, for finite PSPs one usually observes coexistence of synchronized clusters of different sizes, analogously to what reported in Refs. [10,11] for delayed systems. Furthermore, in Ref. [10] the authors found that the average number of coexisting clusters decreases with the delay, somehow analogously to what reported in Ref. [9] for increasing duration of the PSP. As a matter of fact, stable splay states, corresponding to a number of clusters equal to that of the neurons, are observable in the limit of zero delay and instantaneous synapses [12].

The introduction of disorder in the network at the level of either connections or excitability distributions, does not prevent the emergence of COs as shown for systems with delay [13-15] or with finite PSPs [16]. The only case in which COs have been reported in sparse networks in absence of delay and for instantaneous synapses is for quadratic integrate-and-fire (QIF) neurons in a dynamically balanced regime [17].

A common phenomenon observable in inhibitory networks is the progressive silencing (neurons' death) of less excitable neurons induced by the activity of the most excitable ones when the inhibition increases. This mechanism, referred in the literature as Winner Takes All (WTA) with inhibitory feedback [18-20], has been employed to explain attentionactivated competition among visual filters [21], visual discrimination tasks [22,23], as well as the so-called $\gamma$-cycle documented in several brain regions [24]. Furthermore, the WTA mechanism has been demonstrated to emerge in inhibitory spiking networks for heterogeneous distributions of the neural 
excitabilities [25]. However, while in globally coupled networks (GCNs) the increase in synaptic inhibition can finally lead to only few or even only one surviving neuron, in sparse networks (SNs) inhibition can astonishingly promote, rather than depress, neural activity inducing the reactivation of silent neurons [25-27].

Our aim is to analyze in neural networks with delay, the combined effect that synaptic inhibition and different types of disorder produces on neurons' death and reactivation, as well as on the emergence of COs. For GCNs we show that COs can emerge for sufficiently large synaptic inhibition and delay. This despite the fact that the number of active neurons steadily decreases with the inhibition, due to the WTA mechanism. An increase of the heterogeneity in the neural excitabilities promotes both neural's death and the emergence of asynchronous dynamics, these effects are somehow counteracted by considering longer time delays.

In SNs, at sufficiently large synaptic coupling, the current fluctuations induced by the disorder in the presynaptic connections $[13,14]$, are responsible for the firing of inactive neurons. At the same time, these fluctuations desynchronize the neural activity leading to the disappearance of COs. Therefore, in SNs by varying the synaptic coupling one can observe two successive dynamical transitions: one at small coupling from asynchronous to coherent dynamics and another at larger inhibition from COs to asynchronous evolution. Furthermore, we show that the interval of synaptic couplings where COs are observable remains finite in the thermodynamic limit.

The paper is organized as follows: Sec. II is devoted to the introduction of the studied model and of the microscopic and macroscopic indicators employed to characterize its dynamics. The system is analyzed in Sec. III for a globally coupled topology, where the WTA mechanism and the emergence of COs are discussed. In Sec. IV, we study sparse random networks, with emphasis on the role of current fluctuations to induce a rebirth in the neural activity at large synaptic scale as well as their influence on collective behaviors. The combined role of heterogeneity and delay on the dynamical behavior of the system is addressed both for GCNs (Sec. III) and SNs (in Sec. IV). Section V deals with a detailed analysis of the effect of disorder on finite-size networks. Finally, a brief discussion of the reported results can be found in Sec. VI.

\section{MODEL-MICROSCOPIC AND MACROSCOPIC INDICATORS}

We consider a heterogeneous inhibitory random network made of $N$ pulse-coupled leaky integrate-and-fire (LIF) neurons. The evolution of the membrane potential of the $i$ th neuron in the network, denoted by $v_{i}$, is given by

$$
\dot{v}_{i}(t)=a_{i}-v_{i}(t)-\frac{g}{K} \sum_{l=1}^{N} \sum_{n \mid t_{l}^{(n)}<t} S_{i, l} \delta\left(t-t_{l}^{(n)}-t_{d}\right) .
$$

Whenever $v_{i}$ reaches the firing threshold $v_{\theta}=1$ it is instantaneously reset to the resting value $v_{r}=0$ and a $\delta$-spike is emitted at time $t_{i}^{(n)}$ and received by its postsynaptic neighbors after a delay $t_{d}$. The sum appearing in Eq. (1) runs over all the spikes received by the neuron $i$ up to the time $t$. $S_{i, l}$ denotes the connectivity matrix, with entries 1 , whenever a link connects the presynaptic neuron $l$ to the postsynaptic neuron $i$, and 0 , otherwise. For sparse networks we randomly select the nonzero entries of $S_{i, l}$; however, we impose that the number of presynaptic connections is constant and equal to $K$ for each neuron $i$, namely $\sum_{l \neq i} S_{i, l}=K$, since autaptic connections are not allowed. Therefore, for the GCN we have $K=N-1$. The positive parameter $g$ appearing in Eq. (1) represents the coupling strength and the preceding negative sign denotes the inhibitory nature of the synapse. Each neuron is subject to a different suprathreshold input current $a_{i}>v_{\theta}$, representing the contribution both of the intrinsic neural excitability and of the external excitation due to projections of neurons situated outside the considered recurrent network. Heterogeneity in the excitabilities is introduced by randomly drawing $a_{i}$ from an uniform distribution of width $\Delta a=a_{2}-a_{1}$ defined over the interval $\left[a_{1}, a_{2}\right]$. For simplicity, all variables are assumed to be dimensionless.

The microscopic dynamics can be characterized in terms of the interspike interval (ISI) $T_{i \text {,ISI }}$ statistics for each neuron $i$. The statistics is known once the corresponding probability density function (PDF) $P\left(T_{i, \text { ISI }}\right)$ is given, from which it can be obtained the average firing period $T_{i}=\left\langle T_{i, \mathrm{ISI}}\right\rangle$ as well as the coefficient of variation $\mathrm{CV}_{i}=\sigma\left(T_{i, \text { ISI }}\right) /\left\langle T_{i, \text { ISI }}\right\rangle, \sigma\left(T_{i, \text { ISI }}\right)$ being the standard deviation of the ISI distribution. The average firing rate of neuron $i$ is given by $v_{i}=1 / T_{i}$. For the considered heterogeneous distribution of the excitabilities, each isolated neuron is characterized by a different free spiking period, namely $T_{\mathrm{i}, \text { free }}=\ln \left[a_{i} /\left(a_{i}-1\right)\right]$. However, in the network the activity of each neuron is modified by the firing activity of its presynaptic neighbors. In particular, the effective input $\mu_{i}$ to a generic neuron $i$ in the network can be written, within a mean-field approximation, as follows:

$$
\mu_{i}(t)=a_{i}-g v_{A} n_{A}\left(t-t_{d}\right),
$$

where $n_{A}\left(t-t_{d}\right)$ is the percentage of active neurons at time $t-t_{d}$ and $v_{A}$ is the average firing rate of the neurons active at time $t-t_{d}$. A neuron will be supra- or subthreshold depending whether $\mu_{i}(t)$ is larger or smaller than $v_{\theta}$. The percentage of active neurons $n_{A}\left(t_{f}\right)$ in a certain time interval $t_{f}$ is a quantity that we will employ to characterize the network at a microscopic level. Since the firing rate has been defined in terms of the ISI, $n_{A}\left(t_{f}\right)$ is defined as the percentage of neurons that have emitted at least two spikes within a time period $t_{f}$ after discarding a transient corresponding to the emission of $20 \mathrm{~N}$ spikes (we employ these values for all the reported simulations, unless otherwise stated).

To study the collective behavior of the network we introduce an auxiliary field $E_{i}(t)$ for each neuron representing the linear superposition of the received train of spikes filtered opportunely. In particular we filter each spike with a postsynaptic profile having the shape of an $\alpha$-function $p(t)=$ $\alpha^{2} t \exp (-\alpha t)(t>0)$. Therefore, the corresponding effective fields $E_{i}(t)$ can be obtained by integrating the following second order ordinary differential equations:

$$
\ddot{E}_{i}+2 \alpha \dot{E}_{i}+\alpha^{2} E_{i}=\frac{\alpha^{2}}{K} \sum_{l=1}^{N} \sum_{n \mid t_{l}^{(n)}<t} S_{i, l} \delta\left(t-t_{l}^{(n)}-t_{d}\right),
$$

where $\alpha$ represents the inverse pulse width and it is fixed to $\alpha=20$. The integration of the set of ordinary differential 
Eqs. (1) and (3) has been performed in an exact manner by employing a refined event driven technique explained in details in Ref. [15].

The macroscopic dynamics of the network can be analyzed in terms of the mean field

$$
[E(t)]=\frac{1}{N} \sum_{i=1}^{N} E_{i}(t),
$$

which gives a measure of the instantaneous firing activity at the network level. Furthermore, to identify COs it is more convenient to use the standard deviation of the mean field $[E(t)]$ defined as

$$
\sigma([E])=\sqrt{\left\langle[E]^{2}\right\rangle-\langle[E]\rangle^{2}},
$$

where $\langle\cdot\rangle$ indicates the time average.

In general we will always measure either the time average or the standard deviation of $[E]$, hence to avoid overuse of symbols and unless otherwise stated, $\langle E\rangle \equiv\langle[E]\rangle$ and $\sigma(E) \equiv$ $\sigma([E])$.

\section{GLOBALLY COUPLED NETWORK}

First, we will examine how the dynamics of a GCN changes for increasing synaptic coupling strength $g$, for a chosen time delay, and a certain quenched distribution of the neuronal excitability. The results of this analysis are reported in Figs. 1(a)-1(c) for two different system sizes, namely, $N=$ 4000 and $N=8000$. Analogously to what found in absence of delay in Ref. [25], we observe a steady decrease of the value of $n_{A}$ for increasing $g$ and essentially no dependence on the system size. Furthermore, the value of $n_{A}$ is independent of the value of the considered time period $t_{f}$ once a transient time is discarded, as clearly shown in the inset of Fig. 1(a).

For sufficiently small coupling, all the neurons are active (i.e., $n_{A}=1$ ), and the time averaged field $\langle E\rangle$, which is a proxy of the firing activity of the network, presents an almost constant value with few or no fluctuations. This indicates an asynchronous activity [28], as confirmed by the raster plot shown in Fig. 1(d) for $g=0.1$. By increasing the coupling, $n_{A}$ reduces to values below one. This is because now the neuronal population splits in two groups: the winners which are active neurons able to mute the other group, the losers. Usually the winners (the losers) are characterized by high (low) values of the excitability $a_{i}$.Further increases in the inhibition produces a steady decrease of the percentage of active neurons $n_{A}$ as a consequence of the increased inhibitory action of the winners. This in turn induces an enlargement of the family of the losers and an associated decrease in the network activity measured by $\langle E\rangle$ as shown in Fig. 1(b). This is clearly an effect that can be attributed to the WTA mechanism.

In Ref. [10] it has been shown that perfectly synchronized clusters of neurons emerge in homogeneous fully coupled inhibitory networks due to the transmission delay. The presence of disorder (either in the excitability distribution or in the connections) leads to a smearing of the clusters associated to a non perfect synchronization $[15,29,30]$ as we observe in the present case. The smeared clusters are evident in the raster plots reported in Figs. 1(e) and 1(f) for sufficiently large $g$. Furthermore, the partial synchronization among the neurons

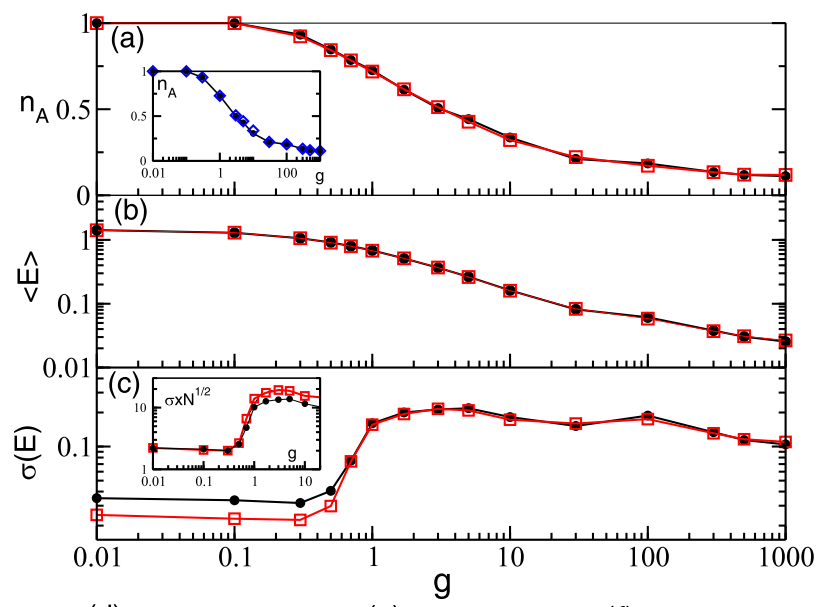

(d)

(e)

(f)

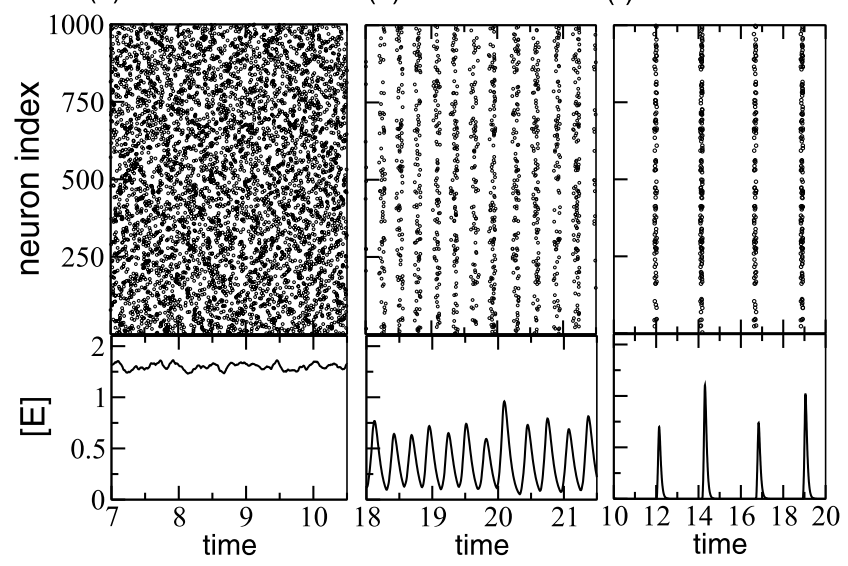

(g)

(h)
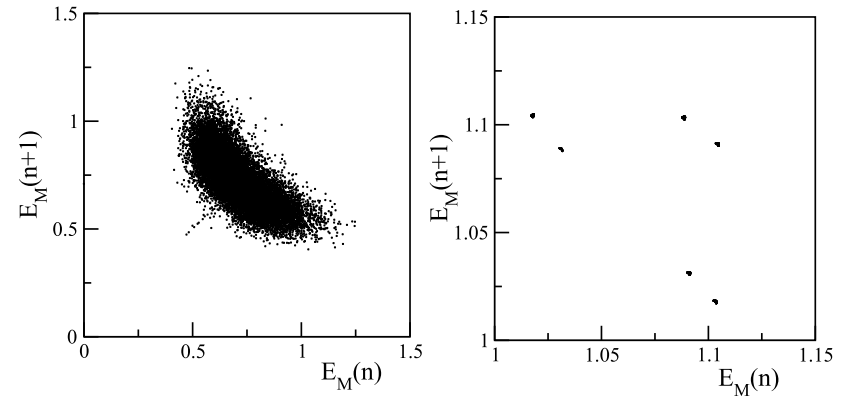

FIG. 1. Winners take all in GCNs: (a) Fraction of active neurons $n_{A}$, (b) time average of the field $\langle E\rangle$, and (c) the corresponding fluctuations $\sigma(E)$ as a function of the strength of the inhibition $g$. In the inset in (a) $n_{A}$ is reported for $N=4000$ and two different time intervals: namely, $t_{f}=5 \times 10^{2}$ time units (black filled circles) and $t_{f}=5 \times 10^{4}$ time units (blue open diamonds). In the inset in (c) $\sigma(E)$ has been multiplied by $\sqrt{N}$. Black filled circles correspond to $N=4000$ while red empty squares represent $N=8000$. (d-f) Raster plots (top) and time traces of the field (bottom) for increasing coupling strength: from the left to the right $g=0.1,3$, and 100 for $N=4000$ (for reasons of clarity in the raster plots only the spikes of 1000 neurons are shown). (g, h) Return maps for the maxima of the field $E_{M}$ for $g=3(\mathrm{~g})$ and $g=100(\mathrm{~h})$ in the case of $N=4000$. Simulations in this figure were obtained after discarding a transient corresponding to $20 \mathrm{~N}$ spikes and calculating the statistics over a time interval $t_{f}=5 \times 10^{2}$ time units, apart for the data shown in panels (g) and (h) where $t_{f}=5 \times 10^{3}$ time units. The data refer to a time delay $t_{d}=0.1$, with $a_{1}=1.2$ and $a_{2}=2.8$, and $\alpha=20$. 
leads (at a macroscopic level) to the emergence of COs in the mean field $[E]$, as shown in the lower panels of Figs. 1(e) and 1(f). In particular, we observe a transition from an asynchronous to collective dynamics, as demonstrated in Fig. 1(c) by reporting $\sigma(E)$ as a function of $g$ for different system sizes. At $g \leqslant 0.5, \sigma(E)$ tends to vanish as $1 / \sqrt{N}$, a typical signature of asynchronous dynamics [31]. For larger values of the coupling strength (namely, $g>0.5), \sigma(E)$ displays a finite value which is independent of the system size, signaling the presence COs. The nature of these oscillations has been previously extensively analyzed in Ref. [29]. In such a study the authors have shown that at intermediate coupling strengths, the collective dynamics is irregular, despite the linear stability of the system, due to the stable chaos mechanisms [32]. This is evident from Fig. 1(e), where the first return map for the maxima of the field $E_{M}(n)$ is reported for $g=3$. In the present analysis we examine much larger coupling strength than in Ref. [32], namely, $g \gg 10$. At these large synaptic strengths we observe that the complexity of the collective dynamics is reduced because the number of active neurons drastically declines, as shown in Fig. 1(a). The few active neurons have a quite limited spread in their excitabilities (namely, $\simeq n_{A} \Delta a$ [33]), thus promoting their reciprocal synchronization. This is also evident from the sharp peaks in the mean-field evolution [see Fig. 1(f)] and from the periodic behavior of the first return map of $E_{M}(n)$, shown in Fig. 1(h).

\section{Role of the heterogeneity and the delay}

To better understand the influence on the dynamics of the parameters entering in the model, we considered different distributions of the excitabilities and different time delays $t_{d}$. Let us consider excitability distributions with different widths $\Delta a=a_{2}-a_{1}$, but with the same average value $[a]=2$, for a fixed value of the delay (namely, $t_{d}=0.1$ ). As shown in Fig. 2(a) and as already demonstrated in absence of delay [25] for $a_{1} \rightarrow v_{\theta}=1$ (corresponding to $\Delta a \rightarrow 2$ in the present case) any arbitrary small amount of inhibition is sufficient to induce neuronal deactivation. However, for increasing values of $a_{1}$ (which amounts to decrease the width $\Delta a$ ) the onset of neuronal deactivation occurs at increasingly larger $g$ values, since larger amount of inhibition are required to silence the neurons with smallest excitability. This also explains why the values of the curves $n_{A}=n_{A}(g)$ decrease for increasing $\Delta a$, as shown in Fig. 2(a).

The average mean field does not present significant modifications with $g$ as seen in Fig. 2(b). This is due to the fact that, from a mean-field perspective, the system is subject to the same average excitability, and hence one does not expect large deviations in the average firing rates. There are only small deviations at very large $g$ where the network with wider dispersion in the excitabilities display slightly smaller firing rates, just because the number of active neurons is drastically decreased. This effect is much more evident in Fig. 2(c), where we can observe that the value of $\Delta a$ significatively affects both the onset and the amplitude of the COs as measured by $\sigma(E)$. This is because the decrease of $n_{A}$ brings to a reduction in the number of partially synchronized neurons, and therefore a reduction of the amplitude of the field's fluctuations.

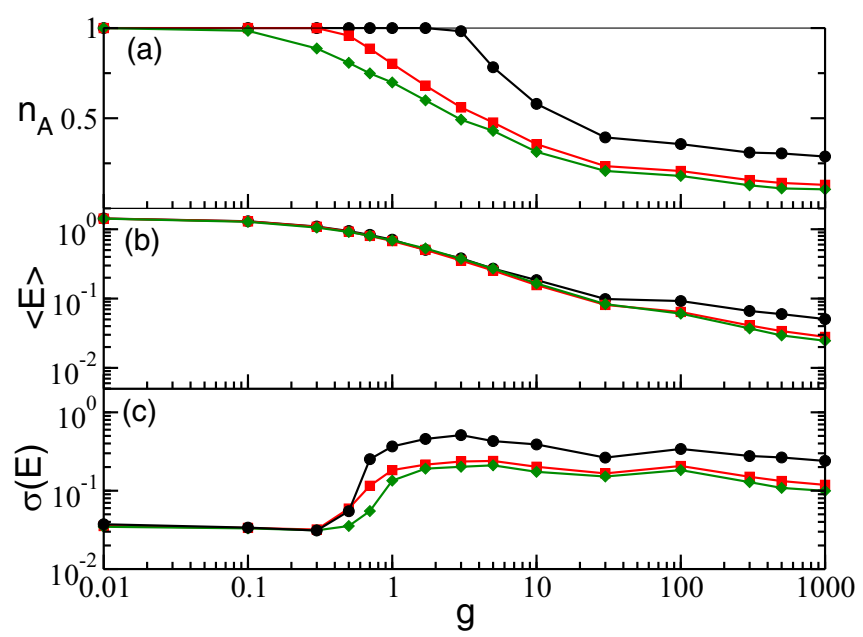

FIG. 2. Relevance of the heterogeneity for the dynamics of GCNs: From top to bottom, (a) fraction of active neurons, (b) average temporal value of the mean field, and (c) average fluctuations of the field $E$, for different strengths of inhibitory connections and several values of heterogeneity. Black circles refer to $\Delta a=0.4$, red squares to $\Delta a=1.2$, and green diamonds to $\Delta a=1.8$. A fixed time delay of $t_{d}=0.1$ is used in this figure. The size of the network is $N=4000$, other parameters as described in the caption of Fig. 1

Let us now consider the influence of the time delay, for a fixed distribution of the excitabilities. From Fig. 3(a), it appears that $n_{A}$ approaches an asymptotic plateau for very large coupling, whose value steadily increases for increasing $t_{d}$. Indeed, for small delays, the survivors reduce to few units, e.g., see the example reported in Fig. 3(a) for $t_{d}=0.005$. This dependence of $n_{A}$ on the delay at large couplings is confirmed

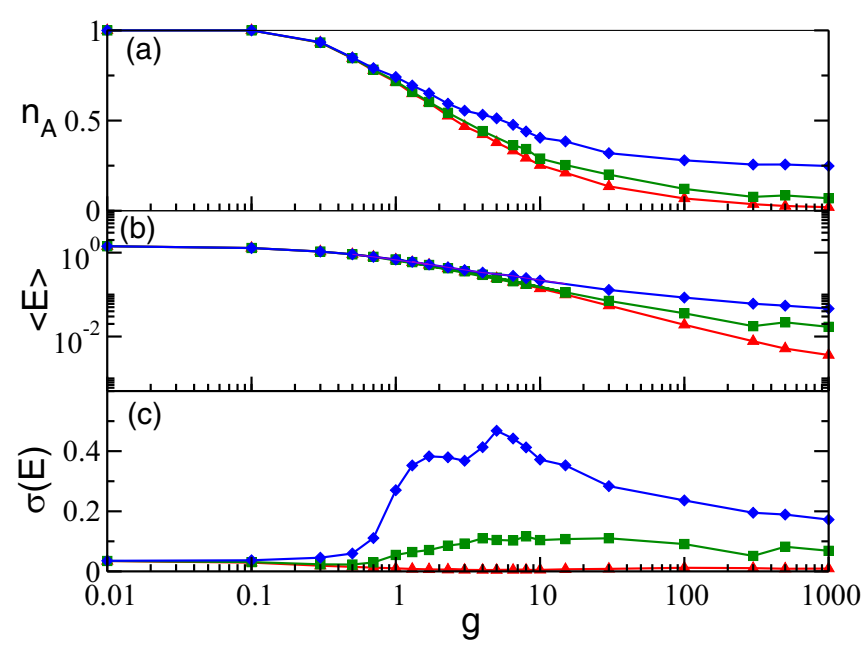

FIG. 3. Relevance of the time delay in GCNs: From top to bottom (a) fraction of active neurons, (b) average temporal value of the mean field, and (c) average fluctuations of the field $E$, for different strengths of inhibitory connections and several values of delay: namely, $t_{d}=0.25$ (blue diamonds), $t_{d}=0.05$ (green squares), and $t_{d}=0.005$ (red triangles). For this figure a fixed heterogeneity distribution with $a_{1}=1.2$ and $a_{2}=2.8$ is used. The size of the network is $N=4000$, other parameters as described in the caption of Fig. 1 
also by the corresponding values of the field $E$ shown in Fig. 3(b). Overall, longer synaptic delays counteract the effect of the heterogeneity and therefore the neural deactivation. As a matter of fact, for $t_{d} \simeq T_{\text {free }} \simeq 1$ the fraction of active neurons is $\simeq 0.67$, even for $\mathrm{g}=1000$ (data not shown).

Furthermore, the delay has a crucial role in the emergence of COs that can be observed already for $t_{d} \geqslant 0.05$ for the parameters considered in Fig. 3(c). Indeed, no collective dynamics have been observed in heterogeneous GCN in absence of delay at any coupling strength [25]. By increasing the delay, we observe larger and larger oscillations in the field $E$, as shown by the curves reported in Fig. 3(c).

These behaviors can be explained by the fact that COs are due to the presence of clusters of neurons at a microscopic level. As shown in Refs. [10,34] for homogeneous systems, and confirmed in Ref. [15] for heterogeneous networks, the average number of clusters $N_{c}$ increases proportionally to the inverse of the delay. For this reason, for small delays we expect to observe an asynchronous state, characterized by $N_{c} \simeq N$. Conversely, for increasing delay, $N_{c}$ decreases and therefore the neurons are more and more synchronized, thus promoting larger collective fluctuations. The increase in the overall synchronization leads to a reduced effective variability in the neuron dynamics which prevents neuronal deactivation. Indeed, disorder promotes deactivation as demonstrated in Ref. [25] in absence of delay, and as shown in Fig. 2(a), where $n_{A}$ is reported for various $\Delta a$ values.

\section{SPARSE NETWORK}

We will now consider the diluted case, i.e. each neuron has now exactly $K<(N-1)$ random presynaptic neighbors. In this case we observe that $n_{A}$ has a nonmonotonic behavior with $g$, as shown in Fig. 4(a) for different values of the in-degree $K$ for a fixed system size, namely, $N=4000$. In particular, we observe that for small coupling, $n_{A}$ decreases in analogy to what reported for the GCN. However, for synaptic couplings larger than a critical value $g_{m}$, the percentage of active neurons increases with $g$. This behavior indicates that for $g>g_{m}$ an increase of the inhibitory coupling can lead to the reactivation of neurons that were inactive for smaller $g$. An analogous behavior has previously been reported in inhibitory sparse networks in absence of delay for conductance based [26] and LIF [25,27] neuronal models. The value of $g_{m}$ grows faster than a power-law with the in-degree $K$, as shown in the inset of Fig. 4(a). In particular, we expect that for $K \rightarrow$ $(N-1)$, i.e., by recovering the fully coupled case, $g_{m} \rightarrow \infty$ and $n_{A}$ converges toward the curve reported in Fig. 1(a).

Analogously to GCNs, the average mean field $\langle E\rangle$ steadily decreases by increasing $g$ indicating that neuronal dynamics slow down for increasing inhibition. This means that for sufficiently large $g$ all the neurons can be reactivated but with a definitely lower firing rate. The dependence of $\langle E\rangle$ with $K$, shown in Fig. 4(b), reveals that for $g<g_{m}$ essentially all curves coincide, while at larger synaptic coupling the smaller is $K$ the larger is the value of the field. This latter behavior is clearly dictated by $n_{A}$ : namely, more neurons are reactivated, higher is the filtered firing rate measured by $\langle E\rangle$.

The mean-field fluctuations $\sigma(E)$ have now a striking different behavior with respect to the GCN. In sparse networks,

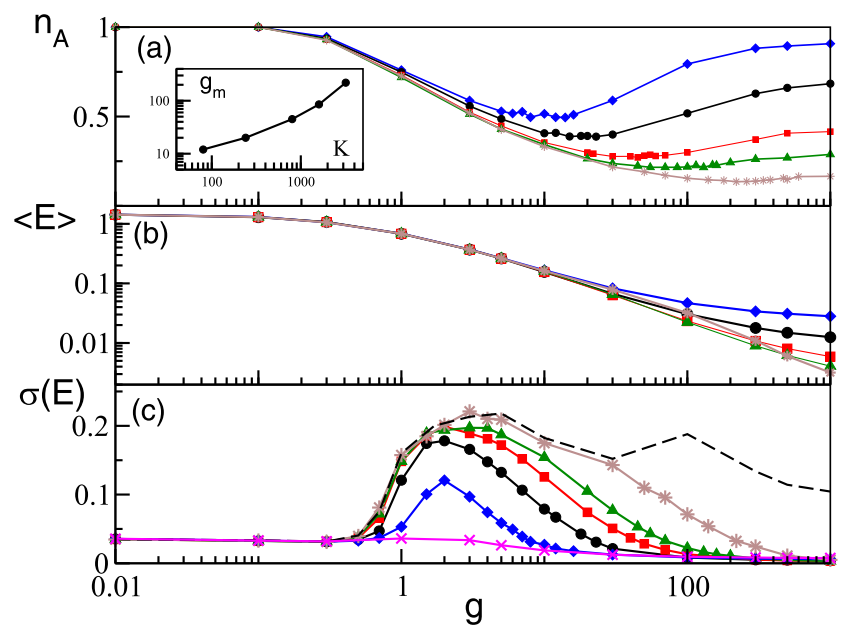

(d)

(e)

(f)

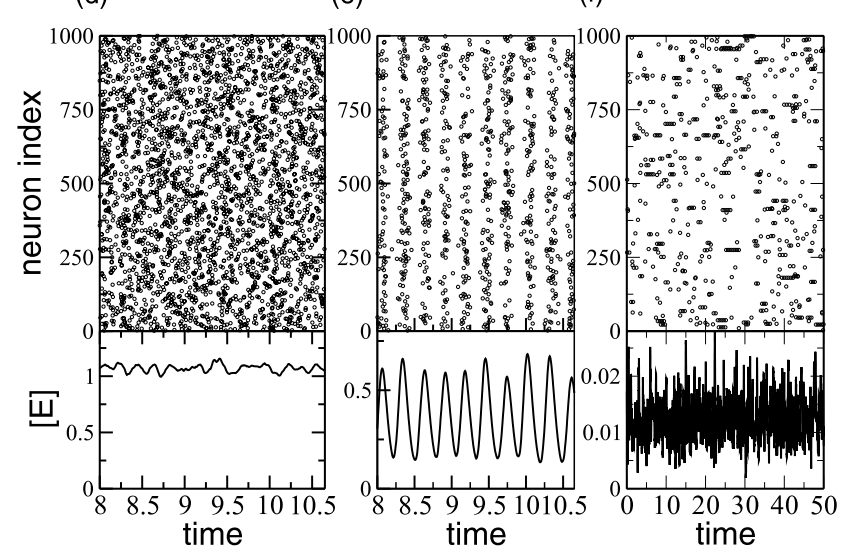

FIG. 4. Reactivation and collective oscillation in SNs: (a) Fraction of active neurons $n_{A}$, (b) average mean field $\langle E\rangle$, and (c) fluctuations $\sigma(E)$ of the mean field as a function of the inhibition. The data refer to a fixed network size $N=4000$ and different in-degrees: namely, $K=40$ (magenta crosses), $K=80$ (blue diamonds), $K=$ 240 (black circles), $K=800$ (red squares), $K=1600$ (green triangles), and $K=3200$ (brown stars). In the inset of (a) the value of $g_{m}$ is plotted versus the in-degree $K$, where $g_{m}$ has been estimated by using a cubic regression in the region of the minimum of $g$. The black dashed curve in (c) refers to the GCN previously reported in Fig. 1(c). (d-f) Raster plot (top) and time course of the mean field [E] (bottom) for $g=0.3$ (d), $g=3$ (e), and $g=1000$ (f). Other parameters as described in the caption of Fig. 1

$\sigma(E)$ displays a maximum at some intermediate $g$ value, while for small and large couplings $\sigma(E)$ tends to vanish, as shown in Fig. 4(c). This suggests that COs are present only at intermediate couplings, while out of this range the dynamics is asynchronous. This is confirmed by the raster plots and the fields' evolution reported in Figs. 4(d)-4(f) for different synaptic couplings. In particular, at small and very large coupling (namely, $g=0.3$ and $g=1000$ ), the instantaneous field $[E]$ is essentially constant [see Figs. 4(d) and 4(f)]. This is a clear indication of asynchronous dynamics. However, the corresponding raster plots differ from one another. Specifically, while for $g=0.3$ the activity is almost homogeneous, for $g=1000$ a sporadic bursting activity characterizes the neuron firing. A detailed analysis of the differences between these two 


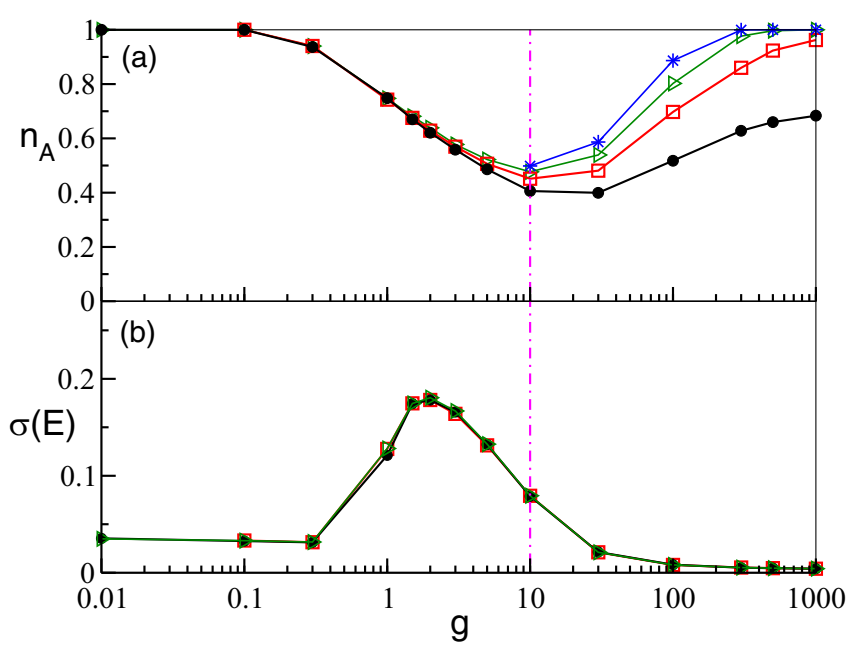

FIG. 5. Finite-time effects in SNs: (a) Fraction of active neurons as a function of inhibition for four different time windows, namely, $t_{f}=5 \times 10^{2}$ (black circles), $t_{f}=5 \times 10^{3}$ (red squares), $t_{f}=5 \times$ $10^{4}$ (green triangles), and $t_{f}=5 \times 10^{5}$ (blue stars). (b) Fluctuations of the mean field as a function of inhibition estimated for the three shortest time windows $t_{f}$ reported in panel (a). The vertical dotdashed magenta line denotes $g_{m}$, as estimated for $t_{f}=5 \times 10^{5}$. The data refer to $N=4000$ and $K=240$, all the other parameters as described in the caption of Fig. 1

asynchronous regimes will be discussed in Sec. IV B. Finally, at intermediate synaptic couplings COs are clearly observable, as testified by the raster plot and the field shown in Fig. 4(e) for $g=3$.

From Fig. 4(c) it is also evident that the onset and the amplitude of the collective dynamics strongly depend on the dilution, measured in terms of the in-degree $K$, and that for $K \rightarrow(N-1)$ the globally coupled behavior is recovered. In particular, smaller is $K$ smaller is the amplitude of the COs and narrower is the synaptic coupling interval where they are observable. These two effects are due to the fact that the disorder in the connectivity distribution increases as $1 / \sqrt{K}$. Therefore, on one side the clusters of partially synchronized neurons, which are responsible for the COs, are more smeared at smaller $K$ thus inducing smaller amplitudes of the oscillations. On the other side, the disorder prevents the emergence of COs, thus the region of existence is reduced at lower $K$. As a matter of fact, for $N=4000$ we start to observe COs only when $K>40$, the critical case $K=40$ is displayed in Fig. 4(c) as magenta crosses. The existence of a critical connectivity for the emergence of collective dynamics is a general feature of sparse networks [17,31].

To understand if the value $t_{f}$ of the time interval over which we measure $n_{A}$ and $\sigma(E)$ has an influence on the observed effects, we also examined the dependence of these two quantities on $t_{f}$ for a fixed size $N$ and in-degree $K$. The results of this analysis are reported in Fig. 5, where it is shown that, on one hand, for $g<g_{m}$ the percentage of active neurons is almost insensible to the considered time window analogously to what observed for the GCN. On the other hand, for $g>g_{m}$, the value $n_{A}$ grows with $t_{f}$ and for sufficiently long times and sufficiently large $g$ all the neurons can be reactivated. However, as shown in Fig. 5(a), the growth of
$n_{A}$ dramatically slows down with increasing $t_{f}$, and we can safely affirm that for $t_{f}>10^{5}$ the further evolution of $n_{A}$ occurs on unrealistically long timescales. For what concerns $\sigma(E)$ finite-time effects are essentially not present, as shown in Fig. 5(b).

\section{A. The role of current fluctuations}

Previous analyses of inhibitory networks in absence of delay [25-27] have clearly shown that the position $g_{m}$ of the minimum of $n_{A}$ marks the transition from a regime dominated by the activity of the suprathreshold neurons (mean driven) to a regime where the most part of the neurons are below threshold and the firing is mainly due to current fluctuations (fluctuation driven) [35].

With the aim of verifying that the origin of neuronal reactivation in the present case is also related to such transition, let us analyze the system from a mean-field perspective. In this framework, the activity of a neuron is completely determined by the average input current and its fluctuations. Let us limit our analysis to the active neurons, since these are the only ones contributing to the network dynamics. In particular, the average effective input to the active neurons can be estimated as follows:

$$
\mu_{A}=I_{A}-g v_{A} n_{A},
$$

where $I_{A}\left(v_{A}\right)$ is the average excitability (firing rate) of the active neurons. Despite the dynamics being fully deterministic, thanks to the sparseness in the connections, each neuron can be seen as subject to $n_{A} K$ uncorrelated Poissonian trains of inhibitory spikes of fixed amplitude $g$ with rate $v_{A}$.Therefore, by following Refs. [13,36] the current fluctuations can be estimated as

$$
\sigma_{A}=g \sqrt{\frac{v_{A} n_{A}}{K}} .
$$

As it can be appreciated from Fig. 6, the theoretical estimations Eqs. (4) and (5) (dashed curves) are in very good agreement with the numerical data for $\mu_{A}$ and $\sigma_{A}$ (filled symbols) over the whole considered range of the synaptic inhibition (corresponding to five orders of magnitude). On the one hand, one observes a steady decrease of $\mu_{A}$ with $g$, which can be understood from its expression Eq. (4), since $v_{A}$ is a quantity monotonically decreasing with the synaptic strength despite the neural reactivation present in SNs. This can be inferred from the behavior of the mean field $[E]$, which is strictly connected to $v_{A}$, reported in Fig. 4(b). On the other hand, the fluctuations of the input currents increase with $g$, thus indicating that in Eq. (5) the growth of $g$ prevails over the decrease of $v_{A}$.

The key result explaining the mechanism behind neural reactivation is reported in Fig. 6: It is clear that $\mu_{A}$ becomes smaller than the firing threshold $v_{\theta}=1$ exactly at $g=g_{m}$, in concomitance with a dramatic growth of the current fluctuations. Hence, for $g>g_{m}$, since all the neurons are on average below threshold, the neural firing is mostly due to current fluctuations and not to the intrinsic excitability of each neuron. For this reason we expect that for large coupling strength, on one side the average firing of the neurons will become slower, as indeed shown in Fig. 4(b), while on the other side 


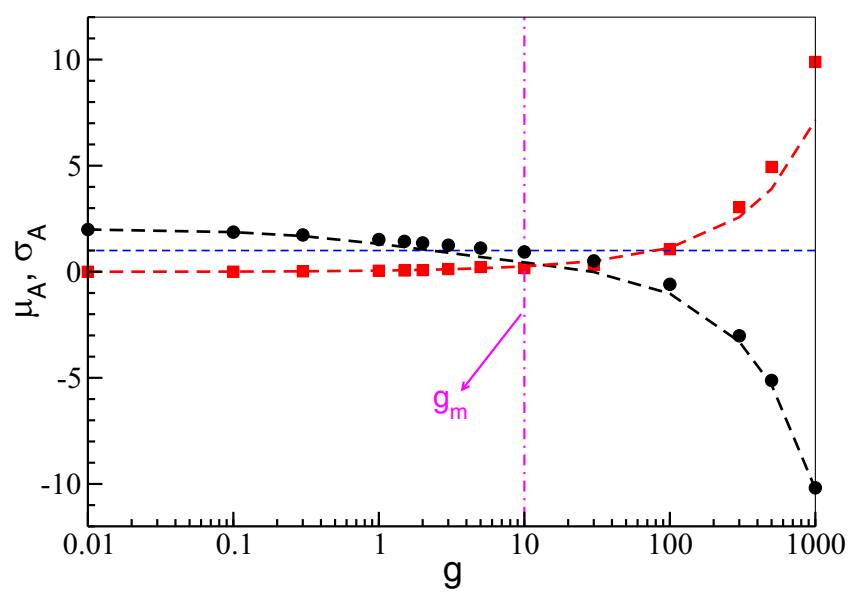

FIG. 6. Mechanism of neural reactivation in SNs: Average $\mu_{A}$ (black) and fluctuations $\sigma_{A}$ (red) of the effective input current in the active subpopulation. The dashed curves are the theoretical estimations [Eqs. (4) and (5)], while the filled symbols represent the numerical estimations. The firing threshold of the LIF neuron is depicted as a blue dashed line. Vertical dot-dashed line line denotes the measured value $g_{m}$. A SN of size $N=4000$ and $K=240$ is considered; all other parameters as described in the caption of Fig. 1.

the fraction $n_{A}$ of firing neurons will increase, thanks to the increase of $\sigma_{A}$ with $g$. Therefore, we can confirm that the occurrence of the minimum in $n_{A}$ signals a transition from a mean driven to a fluctuation driven dynamics, analogously to what found in Ref. [25] in absence of delay.

Nonetheless, in the present case current fluctuations have also a destructive role on the collective dynamics induced by the delay. As it can be inferred from Fig. 5(b) (see also Fig. 11 in the last section), the coherent motion disappears for synaptic coupling larger than $g_{m}$, when the amplitude of the random fluctuations in the input currents becomes sufficiently large to completely desynchronize the neural activity.

\section{B. Characterization of the microscopic dynamics}

The analysis of the microscopic dynamics can clarify the different observed regimes. In particular, we will consider the dynamics of the neurons in a network with $N=4000$ and $K=$ 240 in three typical regimes: namely, in presence of collective motion $\left(0.5<g<g_{m}\right)$, in proximity of the minimum of $n_{A}$ $\left(g \simeq g_{m}=10\right)$ and for very large inhibition $\left(g \gg g_{m}\right)$. For each synaptic coupling we study the distribution of the ISIs, $P\left(T_{\mathrm{ISI}}\right)$, for three representative neurons characterized by high $(\mathrm{H})$, intermediate $(\mathrm{I})$, and low $(\mathrm{L})$ average firing rates.

The results of this analysis are reported in Fig. 7, where we considered $g=2(\mathrm{a}, \mathrm{b}), g=30$ (c), and $g=1000$ (d). In particular, $g=2$ corresponds to the maximum in the amplitude of the COs measured by $\sigma(E)$ (see Fig. 5). For this synaptic coupling the distributions $P\left(T_{\mathrm{ISI}}\right)$ are quite peculiar, being characterized by several peaks separated by a constant time lag $\delta t$. The number of peaks and the value of $\delta t$ increase going from the fastest to the slowest neuron: namely, the time lag $\delta t$ varies from $\sim 0.12$ (F) to $\sim 0.23$ (I) and $\sim 0.25$ (L).

This structure can be traced back to the coherent inhibitory action of clusters of partially synchronized neurons, coarse grained by the collective field $[E]$, on the targeted neuron. This effect is illustrated in Fig. 7(b), where the membrane potential $v_{L}$ of the low firing rate neuron and the corresponding average field $[E]$ are reported during a short time interval. The field $[E]$ displays irregular oscillations due to the clustered activity of the neurons, furthermore it is clear that the occurrence of every local maximum in $[E]$ is in perfect correspondence with a local minimum of $v_{L}$. This means that a spike can only be emitted in correspondence to a local minimum of the field. As a consequence, the $T_{\text {ISI }}$ of neuron (L) should be a multiple of the oscillation period of $[E]$, namely, $\sim 0.25$. The locking with the collective field is progressively less effective for the neurons with higher firing rates [namely, $(\mathrm{I})$ and $(\mathrm{H})$ ] and this reduces the multipeak structure and the value of $\delta t$. Moreover, in this regime dominated by collective inhibitory oscillations, the minimal $T_{\mathrm{ISI}, i}$ for each active neuron is definitely larger than the corresponding free period $T_{\text {free, } i}$. Obviously, the more active the neuron is, the closer to $T_{\text {free, } i}$ is the minimal $T_{\mathrm{ISI}, i}$ [see the inset in Fig. 7(a)].

For larger values of $g \geqslant g_{m}$, the COs vanish and the multipeak structure in $P\left(T_{\text {ISI }}\right)$ disappears accordingly. Moreover, the statistics of the firing times becomes essentially Poissonian as shown in Fig. 7(c). Also, starting from a coupling strength of $g=30$, where no more collective effects are present, the free spiking period of the considered neuron $T_{\text {free, } i}$ appears as the minimal $T_{\mathrm{ISI}, i}$ of the corresponding distribution $P\left(T_{\text {ISI }}\right)$ [see the inset in Fig. 7(c)].

Finally, in the regime of very large $g$, an interesting phenomenon emerges: as shown in Fig. 7(d) for $g=1000$ the ISI distribution displays a large peak at $T_{\text {free, } i}$ and an exponential tail, a typical signature of Poissonian firing. This peculiar structure is due to the bursting activity of the neuron [see also Fig. 4(f)]. Indeed, for this large coupling the firing rate of the presynaptic neurons is very low, therefore the postsynaptic neurons are usually not inhibited and fire with their own free spiking period $T_{\text {free, } i}$. However, whenever they receive sporadically inhibitory kicks of large amplitude $g$, the neurons are silent for a long period necessary for the membrane potential to recover positive values. Furthermore, the Poissonian nature of the distribution of the kick arrival times, is reflected in the long tail of the $P\left(T_{\mathrm{ISI}}\right)$.

Overall, upon increasing inhibition, on one side we observe that the average frequency of neurons steadily decreases, on the other side the neurons tend to fire occasionally at the fastest possible frequency, namely, $1 / T_{\text {free, } i}$. Moreover, increased inhibition produces a steady increase of the variability in the microscopic firing of neurons, as clearly shown in Fig. 8 where we report the ensemble average of the coefficients of variation, [CV]. In particular, we observe upon increasing $g$, a transition from a very regular firing characterized by $[\mathrm{CV}] \simeq 0$ to a dynamics with $[\mathrm{CV}]>1$, which is a signature of multimodal ISI distributions. As shown in Ref. [37], the possible observable $P\left(T_{\mathrm{ISI}}\right)$ distributions in presence of fluctuating inputs are limited only to three types [analogous to the three ones reported in Figs. 7(a), 7(c), and 7(d)], whose occurrence is controlled by the value of the average effective input $\mu$. Furthermore, in Ref. [37] it has been demonstrated that these distributions, for fixed mean $\left\langle T_{\text {ISI }}\right\rangle$ and $\mathrm{CV}$, are independent of the considered neural model, therefore the 

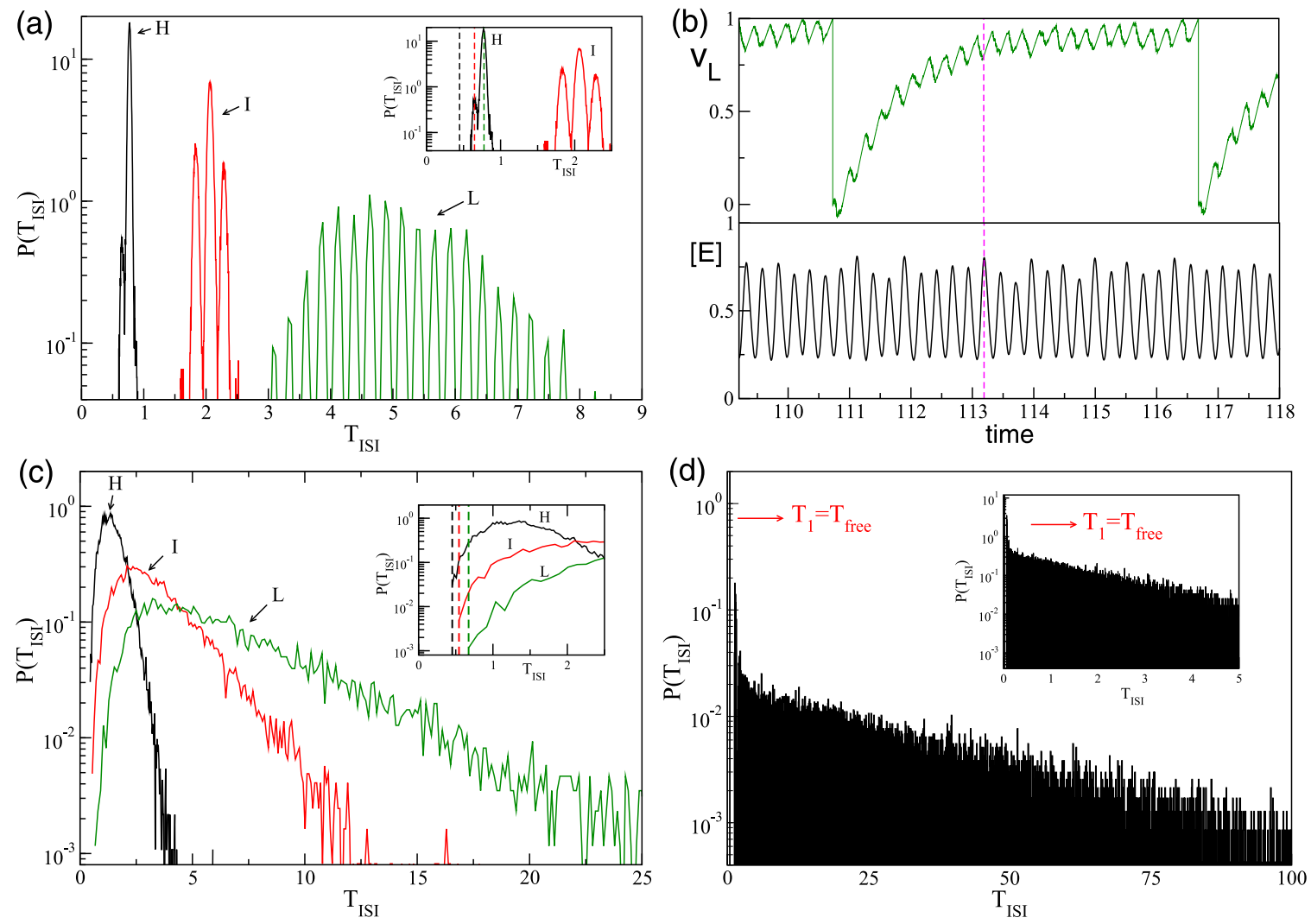

FIG. 7. Microscopic behavior: ISI analysis in SNs. Probability distributions of the ISIs, $P\left(T_{\mathrm{ISI}}\right)$, of representative neurons for increasing values of the coupling strength: namely, $g=2$ (a), $g=30$ (c), and $g=1000$ (d). In (a) and (c) the black, red, and green curves correspond to neurons with high $(\mathrm{H})$, intermediate $(\mathrm{I})$, and low $(\mathrm{L})$ average firing rate, respectively; in the inset is shown a closeup where the free periods of the three neurons (namely, $T_{\text {free, },}, T_{\text {free }, I}$, and $T_{\text {free }, L}$ ) are indicated by vertical dashed lines with the same color code. Note that starting from the coupling strength $g=30$ the value of the free period of the considered active neuron appears as the first channel of the histograms. In (d) the first peak of the histogram, $T_{1}$, corresponds to the period of the considered free neuron $T_{\text {free }}$. In (b) is shown the mechanism originating the multipeak structure in the distributions of panel (a): It is represented on the same time axis an instance of the membrane potential $v_{L}$ of the neuron with low firing rate and of the field $[E]$ (the vertical dashed line marks the locking between local minima of $v_{L}$ and local maxima of $[E])$. Network size $N=4000$ and $K=240$. Other parameters as described in the caption of Fig. 1.

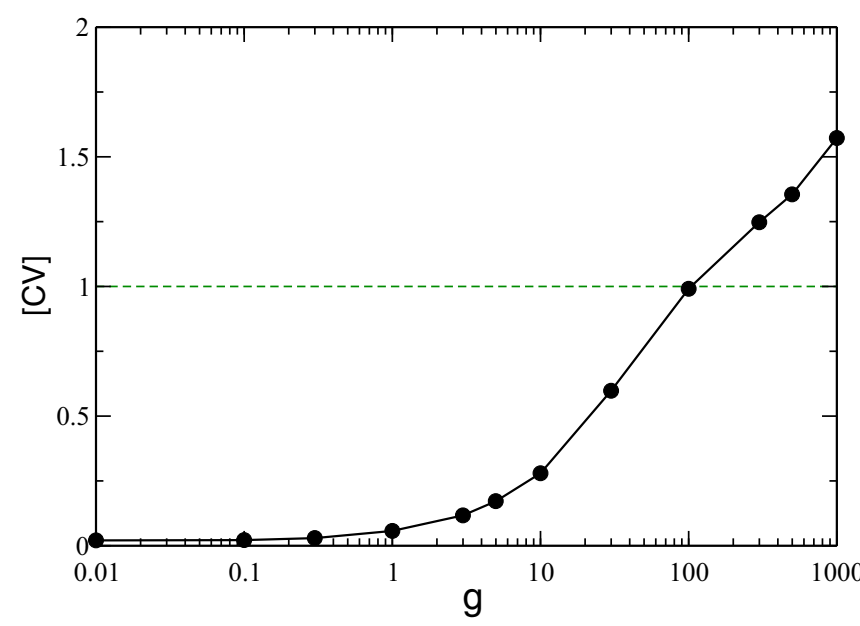

FIG. 8. Microscopic variability of the ISIs in SNs. Ensemble average over all the neurons of the coefficients of variation of the ISIs, [CV], versus the coupling strength $g$. The dashed line signals the value corresponding to a Poissonian statistics, namely, $[\mathrm{CV}]=1$. Network size $N=4000$ and $K=240$, other parameters as described in the caption of Fig. 1. results here reported should be considered as generic ones, not peculiar of LIF networks.

\section{Role of the heterogeneity and of the delay}

Analogously to what done for the GCNs, we analyzed the influence of different excitability distributions as well as of the time delay $t_{d}$ on the dynamics of SNs. The corresponding results are reported in Figs. 9 and 10.

To study the effect of the heterogeneity in the neuronal excitabilities, we choose to keep constant the average $[a]=2$ and to vary $\Delta a$. As discussed in the previous subsections, heterogeneity is necessary for the occurence of the WTA mechanism. Therefore, for small $\Delta a$ the overall deactivationreactivation effect is less evident. This is because the percentage of inactive neurons is much smaller than what reported for larger $\Delta a$ and the complete reactivation of all neurons is obtained at relatively smaller $g$ [see Fig. 9(a)]. Similar to the GCNs, the average network activity measured by $\langle E\rangle$ remains unchanged, because it is mainly dictated by the average synaptic current [see Fig. 9(b)]. Finally, also in this case, the value of $\Delta a$ affects the onset and the amplitude of the collective 


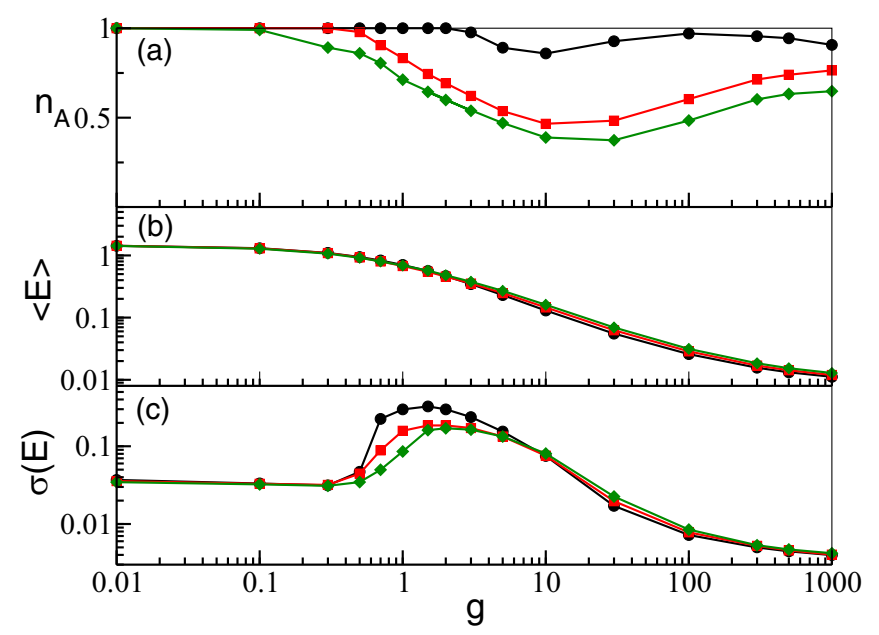

FIG. 9. Relevance of the heterogeneity in SNs. (a) Fraction of active neurons $n_{A}$, (b) time average of the mean field $\langle E\rangle$, (c) fluctuations of the mean field $\sigma(E)$, vs. the synaptic inhibition and for several values of heterogeneity. Namely, $\Delta a=0.4$ (black circles), $\Delta a=1.2$ (red squares), and $\Delta a=1.6$ (green diamonds). In all panels the time delay is set to $t_{d}=0.1$, the network size to $N=4000$ and the in-degree to $K=240$, other parameters as described in the caption of Fig. 1.

motion [see Fig. 9(c)], due to the same mechanism already discussed for GCNs.

Regarding the delay, it is worth to remind that in the globally coupled system, the effects of the synaptic delays were observable in $n_{A}$ and $\langle E\rangle$ only at large inhibition, where the WTA mechanism reduces largely the number of active neurons. This effect is not present in SNs due to the reactivation process occurring at sufficiently large $g$ [see Figs. 10(a)

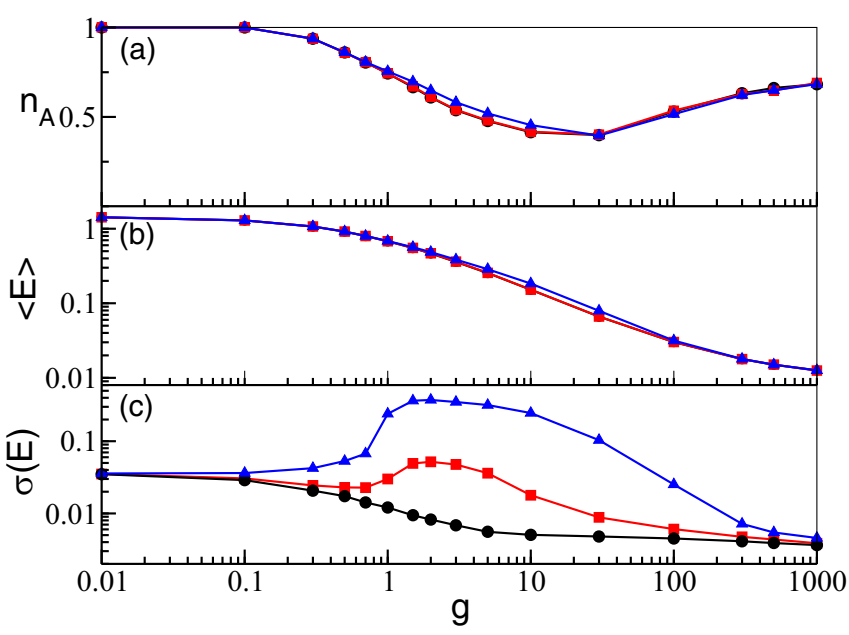

FIG. 10. Relevance of time delay is SNs. (a) Fraction of active neurons $n_{A}$, (b) time average of the mean field $\langle E\rangle$, (c) fluctuations of the mean field $\sigma(E)$, vs. $g$ and different values of time delay. Namely, $t_{d}=0.005$ (black circles), $t_{d}=0.05$ (red squares), and $t_{d}=0.25$ (blue diamonds). For this figure a fixed heterogeneity distribution with $\Delta a=1.6$ is used. In all the panels a fixed value of $N=4000$ and $K=240$ are employed, other parameters as described in the caption of Fig. 1.

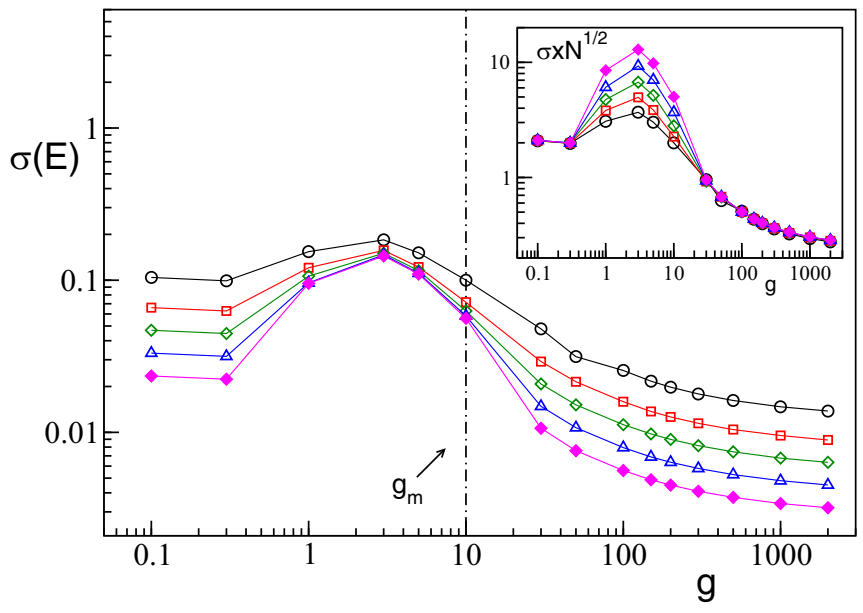

FIG. 11. Finite-size scaling of the mean-field fluctuations in SNs. Standard deviation of the mean field, $\sigma(E)$, as a function of inhibition for networks with $K=150$ and increasing size. Namely, $N=400$ (black circles), $N=1000$ (red squares), $N=2000$ (green diamonds), $N=4000$ (blue triangles), and $N=8000$ (magenta diamonds). The vertical dot-dashed line marks $g_{m}$. In the inset the standard deviation has been rescaled with the system size $N$. Each point in the figure and in the inset is the average over 10 realizations of the disorder. Other parameters as described in the caption of Fig. 1.

and 10(b)]. However, similar to the GCNs, the collective activity can emerge only for sufficiently long delays, namely, $t_{d}>0.005$, and the amplitude of the COs, measured by $\sigma(E)$ increases with $t_{d}$ as shown in Fig. 10(c).

\section{FINITE-SIZE EFFECTS}

In this section we report a detailed analysis of the effects of the disorder on finite-size networks. In GCNs the only source of disorder is associated to the distribution of the excitabilities, while in SNs, the disorder is due also to the random distribution of the connections. In both cases we consider for each system size 10 different network realizations, which implies different excitability and connectivity distributions.

Let us first consider the field $E$ and its fluctuations $\sigma(E)$. Similar to what was reported for the GCNs [see Fig. 1(b)], for the SNs the average value of $\langle E\rangle$ does not depend on $N$ (data not shown). Instead, the mean-field fluctuations strongly depend on the size $N$, as shown also for the GCNs in Fig. 1(c). In particular, for SNs we report $\sigma(E)$ as a function of $g$ in Fig. 11 for a fixed in-degree $K=150$ for system sizes ranging from $N=400$ to $N=8000$. From Fig. 11 (and the inset) it is clear that for $g \leqslant 0.3$ and $g \geqslant 30, \sigma(E) \propto N^{-1 / 2}$, indicating that in the thermodynamic limit the dynamics is asynchronous for small and large couplings. For intermediate values of $g$ (namely, $0.3<g<30$ ) $\sigma(E)$ saturates, for sufficiently large $N$, to an asymptotic finite value, thus showing clearly the persistence of the collective behavior in the thermodynamic limit. The saturation is already observable for $N \geqslant 4000$ for $1 \leqslant g \leqslant 5$. Therefore, we can safely affirm that in the thermodynamic limit COs are present within a finite interval of the synaptic couplings and that their amplitude does not diverge. Furthermore, the width of the interval is determined by the value of $K$ [as shown in Fig. 4(c)] but not by the size 

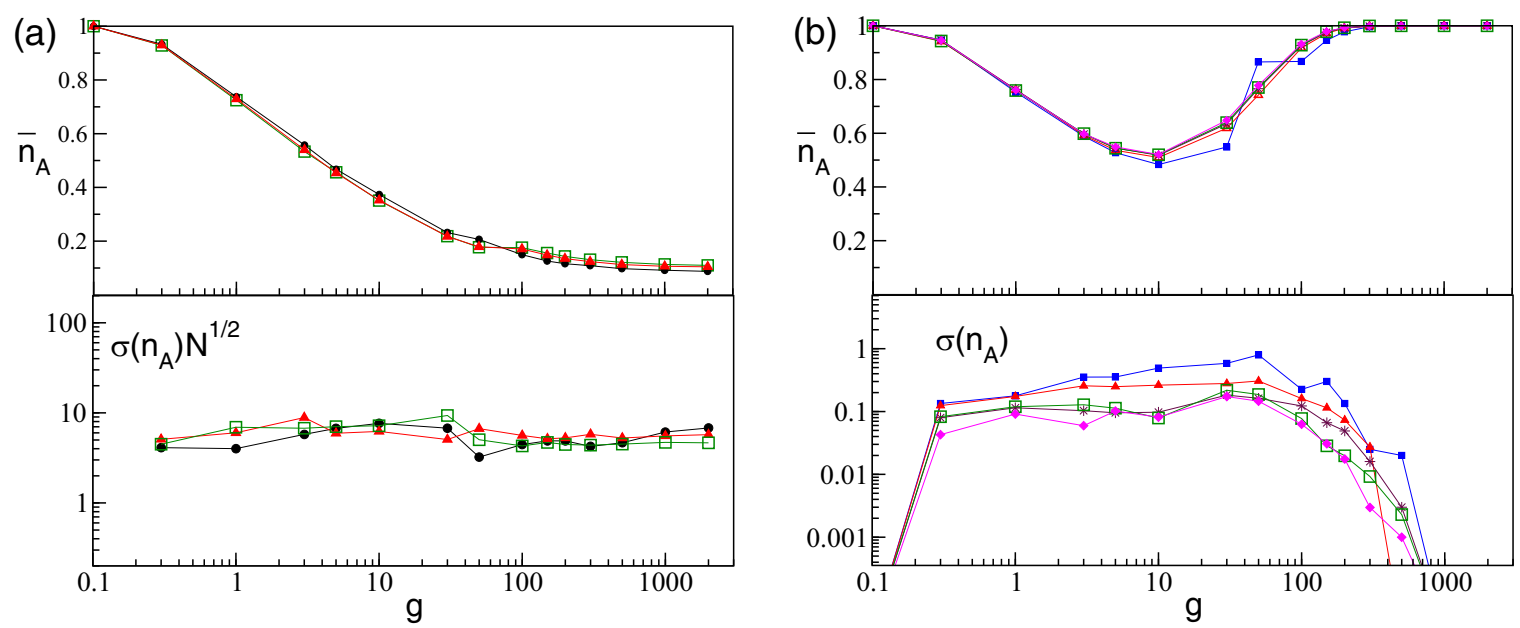

FIG. 12. Fraction of active neurons: Finite-size effects. Panel (a) refers to GCNs, while panel (b) refers to SNSs with $K=150$, for different network sizes: namely, $N=200$ (black circles), $N=400$ (blue squares), $N=1000$ (red triangles), $N=2000$ (maroon stars), $N=4000$ (green squares), and $N=8000$ (magenta diamonds). The panels display the average $\overline{n_{A}}$ (top) and the standard deviation $\sigma\left(n_{A}\right)$ (bottom) of the fraction of active neurons versus the coupling strength $g$. The average and fluctuations have been measured over 10 different realizations of the disorder in the network for each value of $g$ and $N$. In the case of GCNs the standard deviation has been multiplied by the square root of the system size. Remaining parameters as described in the caption of Fig. 1.

of the network $N$. Furthermore, for SNs with sufficiently long delay we have two phase transitions: from asynchronous to collective behavior (at small coupling) and from collective to asynchronous dynamics (at large $g$ ).

Let us now consider the effect of the realizations of disorder on the percentage of active neurons. In particular, in Fig. 12 we report the average, $\bar{n}_{A}$, and the standard deviation, $\sigma\left(n_{A}\right)$, of the fraction of active neurons obtained for 10 different network realizations for increasing $N$ both for GCNs an SNs. As a general remark we observe that $\bar{n}_{A}$ is not particularly sensible to the system size, apart for really small sizes $(N<500)$ in the $\mathrm{SN}$ case [see the upper panels in Figs. 12(a) and 12(b)]. The case of small network sizes for SNs will be discussed later in this section.

For the GCNs, we observe, as expected, a decrease of $\sigma\left(n_{A}\right)$ as $N^{-1 / 2}$ with the system size, as clearly evident from the lower panel in Fig. 12(a). Moreover, we observe that $\sigma\left(n_{A}\right)$ is essentially constant over the whole coupling range, apart for very small coupling strength, $g \leqslant 0.1$ (not shown in the figure), where due to the essentially uncoupled dynamics of the neurons $\sigma\left(n_{A}\right)=0$ for every $N$. The behavior is quite different for SNs as shown in Fig. 12(b) for networks with $K=$ 150. As a general remark we observe that whenever $\bar{n}_{A} \rightarrow 1$ (i.e., for $g<0.3$ and $g>1000$ ) the fluctuations vanish and $\sigma\left(n_{A}\right)$ exhibit finite values in the range of synaptic strength where $\bar{n}_{A}<1$. Furthermore, for increasing $N$ the values of $\sigma\left(n_{A}\right)$ saturate toward an asymptotic profile. Therefore, the fluctuations will persist even in the thermodynamic limit, in agreement with the results reported in Ref. [31], and they assume an almost constant value $\left[\sigma\left(n_{A}\right) \simeq 0.1\right]$ in the range of existence of COs (namely, $0.3<g<30$ ).

The sparseness of the network can give rise to striking effects for small system size, as it is shown in Fig. 13. In particular, in Fig. 13(a) we report the values of $n_{A}$ for 2000 different realizations of the network for $N=200$ and $K=$ 150 . For small coupling strength, namely $g \leqslant 50$, we observe that the distribution of $n_{A}$ values has a single peak centered around the average $\bar{n}_{A}$. While, for larger coupling strength the distribution reveals two distinct peaks: one associated to the typical dynamics of a sparse network at large $g$ (i.e., neural reactivation) and one to the typical dynamics of a GCN (i.e., the WTA mechanism). Thus, rendering the definition of $\bar{n}_{A}$ quite questionable. As a matter of fact, for $g>50$ we estimated two distinct averages for each $g$, one based on the $n_{A}$ values larger than $n_{A}=0.2$ and one on the smaller values, these are reported as red lines in Fig. 13(a). We observe this coexistence of two different type of dynamics also by considering different initial conditions for a fixed disorder realization (data not shown).

The group of networks with very low $n_{A}$ are characterized by a peculiar dynamics where only few neurons remain active. An example of this dynamics for $g=200$ is reported in Fig. 13(b), this state is also indicated by a green arrow in Fig. 13(a). The corresponding raster plot reveals, after a short transient, the convergence toward a dynamical state where only few neurons survive (namely, three in this case), while the rest of the network becomes silent. The interesting aspect is that these three neurons are completely uncoupled among them and their activity is sufficient to silence all the rest of the neurons. The microscopic analysis reveals that the three neurons have high intrinsic excitability (but not necessarily the highest) and that the ensemble of their postsynaptic neurons correspond to the whole network, apart themselves.

The reported effects, i.e., the coexistence of different dynamics as well as the existence of states made of totally uncoupled neurons, disappear increasing the system size. As a matter of fact these effects are already no more observable for $N=400$. Indeed, an analysis of small networks reveals that the peculiar states characterized by few neurons (two or three) with high excitabilities (namely, among the top 20\% neurons) uncoupled among them but with postsynaptic connections projecting over the whole network becomes extremely rare already when $N / K \simeq 2$. 

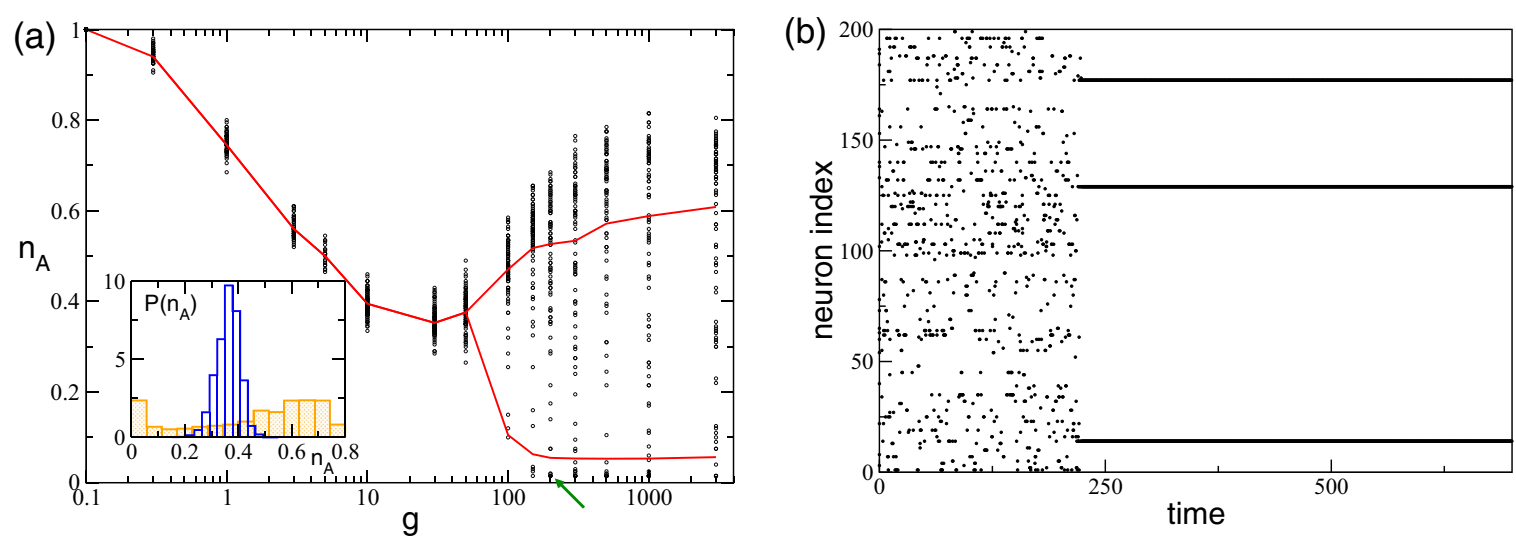

FIG. 13. Effects of the realizations of disorder on small SNs. (a) The plot reports the values of $n_{A}$ obtained for 2000 different realizations of the network for each value of the coupling strength $g, 80$ randomly chosen realizations are shown as black symbols for each $g$. The red lines are averages $\bar{n}_{A}$ over the realizations of the disorder: for $0.1 \leqslant g \leqslant 50$ the average is computed over the total number of realizations, while for coupling strengths larger than $g=50$ the averages are performed by considering two groups of networks as explained in the text. Inset: Probability distributions of $n_{A}$ for $g=30$ (blue histogram) and $g=300$ (orange histogram). (b) The raster plot displays an example of a the dynamical evolution of a peculiar state obtained for large coupling (here $g=200$ ), which is indicated in panel (a) by a green arrow. Data refer to $N=200$ and $K=150$, other parameters as described in the caption of Fig. 1 .

\section{CONCLUDING REMARKS}

In this paper we have clarified how in an inhibitory spiking network the introduction of various ingredients, characteristic of real brain circuits, like delay in the electric signal transmission, heterogeneity of the neurons, and random sparseness in the synaptic connections, can influence the neural dynamics.

In particular, we have studied at a macroscopic and microscopic level the dynamics of heterogeneous inhibitory spiking networks with delay for increasing synaptic coupling. In GCNs the heterogeneity is responsible for neuron's death via the WTA mechanism, while the delay allows for the emergence of COs beyond a critical coupling strength. Furthermore, we have shown that the increase in the delay favors the overall collective dynamics (synchronization) in the system, thus reducing the effective variability in the neuron dynamics. Therefore, longer delays counteract the effect of heterogeneity in the system, which promotes neural deactivation and asynchronous dynamics. For GCNs and SNs, sufficiently long time delays are needed to sustain COs. The required time delays are of the order of $1-5 \mathrm{~ms}$, by assuming a membrane time constant of $20 \mathrm{~ms}$, these can be considered as realistic values, since they are comparable to axonal conduction delays measured in the brain [38].

In SNs by increasing the coupling we observe a passage from a mean driven to a fluctuation driven dynamics induced by the sparseness in the connections. We have a transition from a regime where the neurons are on average suprathreshold to a phase where they are on average below-threshold and their firing is induced by large fluctuations in the currents. This transition is signaled by the occurrence of a minimum in the value of the fraction of active neurons as a function of the inhibitory coupling. Therefore, we can affirm that we pass from a regime dominated by the WTA mechanism, to an activation regime controlled by fluctuations, where all neurons are finally firing but with firing rates definitely lower than those dictated by their excitabilities [25]. However, the fluctuations desynchronize the neural dynamics: the COs emerging at small coupling, due to the time delay, disappear at large coupling when current fluctuations become predominant in the neural dynamics.

Finite-size analysis confirm that in SNs we have two phase transitions that delimit the finite range of couplings where COs are observable. Outside this range the dynamics is asynchronous; however, we have two different kinds of asynchronous dynamics at low and high coupling. At low coupling, we observe a situation where the firing variability of each neuron is quite low and essentially the active neurons behave almost independently. At large coupling, the variability of the firing activity is extremely large, characterized by a bursting behavior at the level of single neurons. Due to the sparseness and the low activity of the fluctuations activated presynaptic neurons, each neuron is subject to low rate Poissonian spike trains of PSPs of large amplitude. Therefore, the neurons are active for a long time and unaffected by the other neurons; however, when they receive large inhibitory PSPs they remain silent for long periods.

It should be remarked that the role of the heterogeneity is fundamental for the occurrence of these two phase transitions, indeed in inhibitory sparse delayed networks made of integrate-and-fire neurons with no intrinsic excitability only the transition from asynchronous to collective dynamics at low synaptic coupling is present [13]. Furthermore, it has been recently shown that for LIF balanced networks with homogeneous excitability the collective dynamics depends on how the thermodynamic limit is taken. In particular, for sparse (massive) networks where the connectivity $K$ is independent of (proportional to) the system size $N$ only asynchronous states (collective irregular oscillations) are observable for any coupling strength [39-41]. In the present paper we limited our analysis to SNs, future work should be devoted to characterize the transitions among different dynamical states in massive networks. 
The observed transition from COs to asynchronous dynamics observable at large synaptic strength can have some biological plausibility in terms of a sort of homeostatic mechanism able to prevent abnormal synchronization in inhibitory circuits. Usually inhbitory plasticity has been experimentally revealed for inhibitory-excitatory connections [42]. However, there are conjectures supported by preliminary evidences that in the Striatum synaptic plastic effect can be present in purely inhibitory networks, as the one involving fastspiking interneurons and medium spiny neurons (MSNs) or even MSNs with other MSNs [43]. In particular, during COs we could expect Hebbian synaptic reinforcement among the recurrently coupled neurons due to some form of plasticity among the inhibitory neurons [42]. This reinforcement will drive the system toward the asynchronous regime, where it will desynchronize. As a consequence the synapses will weaken leading back the network toward the $\mathrm{CO}$ regime. Similar phenomena of decoupling through synchrony have been reported for networks of pyramidal (excitatory) neurons with spike-timing-dependent plasticity in presence of axonal delays [44] or for LIF networks in absence of delays [45]. This mechanism will maintain the network at the border between randomness and partial synchronization, thus favoring the emergence of almost synchronized clusters of cells, which is typical feature of the activity of the MSNs in the striatum [46]. Therefore, it will be worth to examine in future works the dynamics of our network in presence of synaptic plasticity and to understand the role played by the delay in this context.
It has been shown that heterogeneity and noise can increase the information encoded by a population counteracting the correlation present in neuronal activity [47-51]. However, it remains to be clarified how disorder (neural heterogeneity and randomness in the connections) and delay should combine to enhance information encoding. The results reported in this paper can help in understanding the influence of delay and disorder on the dynamics of neural circuits and therefore on their ability to store and recover information.

\section{ACKNOWLEDGMENTS}

The authors acknowledge N. Brunel, V. Hakim, and S. Olmi, for enlightening discussions. A.T. has received partial support by the Excellence Initiative I-Site Paris Seine (Grant No. ANR-16-IDEX-008), by the Labex MME-DII (Grant No. ANR-11-LBX-0023-01), and by the ANR Project ERMUNDY (Grant No. ANR-18-CE37-0014) all funded by the French programme "Investissements d'Avenir." D.A.-G. has received financial support from Vicerrectoria de Investigaciones-Universidad de Cartagena (Project No. 085-2018) and from CNRS for a research period at LPTM, UMR 8089, Université de Cergy-Pontoise, France. The work has been partly realized at the Max Planck Institute for the Physics of Complex Systems (Dresden, Germany) as part of the activity of the Advanced Study Group 2016/17 "From Microscopic to Collective Dynamics in Neural Circuits."
[1] Y. Ben-Ari, Trends Neurosci. 24, 353 (2001).

[2] P. Jonas and G. Buzsaki, Scholarpedia 2, 3286 (2007).

[3] M. A. Whittington, R. Traub, N. Kopell, B. Ermentrout, and E. Buhl, Int. J. Psychophysiol. 38, 315 (2000).

[4] G. Buzsaki, Rhythms of the Brain, 1st ed. (Oxford University Press, New York, 2006).

[5] J. A. Cardin, M. Carlén, K. Meletis, U. Knoblich, F. Zhang, K. Deisseroth, L.-H. Tsai, and C. I. Moore, Nature 459, 663 (2009).

[6] V. S. Sohal, F. Zhang, O. Yizhar, and K. Deisseroth, Nature 459, 698 (2009).

[7] G. Chen, Y. Zhang, X. Li, X. Zhao, Q. Ye, Y. Lin, H. W. Tao, M. J. Rasch, and X. Zhang, Neuron 96, 1403 (2017).

[8] J. A. Cardin, Trends Neurosci. 41, 689 (2018).

[9] C. van Vreeswijk, Phys. Rev. E 54, 5522 (1996).

[10] U. Ernst, K. Pawelzik, and T. Geisel, Phys. Rev. Lett. 74, 1570 (1995).

[11] J. Friedrich and W. Kinzel, J. Comput. Neurosci. 27, 65 (2009).

[12] R. Zillmer, R. Livi, A. Politi, and A. Torcini, Phys. Rev. E 74, 036203 (2006).

[13] N. Brunel and V. Hakim, Neural. Comput. 11, 1621 (1999).

[14] N. Brunel, J. Comput. Neurosci. 8, 183 (2000).

[15] A. Politi and S. Luccioli, in Network Science: Complexity in Nature and Technology, edited by E. Estrada, M. Fox, D. J. Higham, and G.-L. Oppo (Springer, London, 2010), p. 217.

[16] D. Golomb and D. Hansel, Neural Comput. 12, 1095 (2000).

[17] M. di Volo and A. Torcini, Phys. Rev. Lett. 121, 128301 (2018).
[18] R. Coultrip, R. Granger, and G. Lynch, Neural Netw. 5, 47 (1992).

[19] T. Fukai and S. Tanaka, Neural Comput. 9, 77 (1997).

[20] L. Itti and C. Koch, Nat. Rev. Neurosci. 2, 194 (2001).

[21] D. K. Lee, L. Itti, C. Koch, and J. Braun, Nat. Neurosci. 2, 375 (1999).

[22] X.-J. Wang, Neuron 36, 955 (2002).

[23] K.-F. Wong and X.-J. Wang, J. Neurosci. 26, 1314 (2006).

[24] P. Fries, D. Nikolić, and W. Singer, Trends Neurosci. 30, 309 (2007).

[25] D. Angulo-Garcia, S. Luccioli, S. Olmi, and A. Torcini, New J. Phys. 19, 053011 (2017).

[26] A. Ponzi and J. R. Wickens, PLoS Comput. Biol. 9, e1002954 (2013).

[27] D. Angulo-Garcia, J. D. Berke, and A. Torcini, PLoS Comput. Biol. 12, e1004778 (2015).

[28] S. Olmi, A. Politi, and A. Torcini, J. Math. Neurosci. 2, 12 (2012).

[29] S. Luccioli and A. Politi, Phys. Rev. Lett. 105, 158104 (2010).

[30] R. Zillmer, N. Brunel, and D. Hansel, Phys. Rev. E 79, 031909 (2009).

[31] S. Luccioli, S. Olmi, A. Politi, and A. Torcini, Phys. Rev. Lett. 109, 138103 (2012).

[32] A. Politi and A. Torcini, in Nonlinear Dynamics and Chaos: Advances and Perspectives, edited by M. Thiel, J. Kurths, M. C. Romano, G. Károlyi, and A. Moura (Springer, Berlin, 2010), pp. 103-129. 
[33] The winners are the neurons with higher values of the excitability. In the case of a uniform distribution defined in the interval $\left[a_{1}: a_{2}\right]$ the smallest excitability of an active neuron is $a_{S}=a_{2}-n_{A} \Delta a$. Therefore, the spread of the excitabilities of the active neuron is given by $a_{2}-a_{S}=n_{A} \Delta a$.

[34] U. Ernst, K. Pawelzik, and T. Geisel, Phys. Rev. E 57, 2150 (1998).

[35] A. Renart, R. Moreno-Bote, X.-J. Wang, and N. Parga, Neural. Comput. 19, 1 (2007).

[36] H. C. Tuckwell, Introduction to Theoretical Neurobiology: Volume 2, Nonlinear and Stochastic Theories (Cambridge University Press, Cambridge, 2005), Vol. 8.

[37] S. Ostojic, J. Neurophysiol. 106, 361 (2011).

[38] H. A. Swadlow and S. G. Waxman, Scholarpedia 7, 1451 (2012).

[39] S. Ostojic, Nat. Neurosci. 17, 594 (2014).

[40] E. Ullner, A. Politi, and A. Torcini, Chaos: Interdiscip. J. Nonlinear Sci. 28, 081106 (2018).
[41] A. Politi, E. Ullner, and A. Torcini, Eur. Phys. J. Spec. Top. 227, 1185 (2018).

[42] D. M. Kullmann, A. W. Moreau, Y. Bakiri, and E. Nicholson, Neuron 75, 951 (2012).

[43] A. C. Kreitzer and R. C. Malenka, Neuron 60, 543 (2008).

[44] E. V. Lubenov and A. G. Siapas, Neuron 58, 118 (2008).

[45] K. Mikkelsen, A. Imparato, and A. Torcini, Phys. Rev. Lett. 110, 208101 (2013).

[46] A. Ponzi and J. Wickens, J. Neurosci. 30, 5894 (2010).

[47] M. Shamir and H. Sompolinsky, Neural Comput. 18, 1951 (2006).

[48] K. Padmanabhan and N. N. Urban, Nat. Neurosci. 13, 1276 (2010).

[49] A. S. Ecker, P. Berens, A. S. Tolias, and M. Bethge, J. Neurosci. 31, 14272 (2011).

[50] J. F. Mejias and A. Longtin, Front. Comput. Neurosci. 8, 107 (2014).

[51] J. F. Mejias and A. Longtin, Phys. Rev. Lett. 108, 228102 (2012). 